\title{
In vitro evaluation of Staphylococcus aureus, Pseudomonas aeruginosa, and Candida albicans polymicrobial biofilm growth on synthetic surgical implant materials
}

\author{
Bridget A Colgan ${ }^{1}$, Brooke A Pati ${ }^{1}$, Wendy E Kurata ${ }^{2}$, Timothy S Horseman ${ }^{2}$ and Lisa M Pierce ${ }^{2 *}$ \\ ${ }^{1}$ Department of General Surgery, Tripler Army Medical Center, 1 Jarrett White Road, Honolulu, Hawaii, 96859, USA \\ ${ }^{2}$ Department of Clinical Investigation, Tripler Army Medical Center, 1 Jarrett White Road, Honolulu, Hawaii, 96859, USA
}

\begin{abstract}
Biofilms are a known important contributor to the infectious complications associated with prosthetic mesh implantation. Previous studies have demonstrated the formation of monomicrobial biofilms on surgical mesh materials by different bacterial species in vitro, but a paucity of data exists examining polymicrobial biofilm formation by antibiotic-resistant organisms, which may be more clinically relevant. Therefore, the aim of this study was to evaluate the ability of three clinical bacterial and fungal isolates to form mixed-species and single-species biofilms on five synthetic implant materials in vitro, including monofilament polypropylene, monofilament polyester, multifilament polyester, monofilament polytetrafluoroethylene (PTFE), and silicone. Methicillin-resistant Staphylococcus aureus (MRSA), multidrug-resistant Pseudomonas aeruginosa, and Candida albicans (alone or in combination) were inoculated into culture medium containing meshes and allowed to attach and propagate into mature biofilms for 48 hours at $37^{\circ} \mathrm{C}$. Additional samples were inoculated with Staphylococcus epidermidis for comparison. Biofilm biomass and the number of viable cells were quantified by crystal violet staining and colony forming units (CFU) counting, respectively. Images of polymicrobial biofilms were obtained using fluorescence microscopy of $\mathrm{FM}^{\circledast} 1-43$-stained meshes. All materials investigated were capable of harboring biofilms, but the relative abundance was dependent upon both material and organism type. Overall, PTFE meshes exhibited the highest levels of polymicrobial biofilm formation. When comparing monomicrobial biofilms, Pseudomonas aeruginosa demonstrated greater biomass than MRSA and Staphylococcus epidermidis on PTFE, polypropylene, and monofilament polyester, while MRSA and Staphylococcus epidermidis biomass did not differ significantly among mesh types. Thus MRSA, Pseudomonas aeruginosa, and Candida albicans can cooperatively form mature biofilms on surgical implant materials, but different mesh constructs vary in their susceptibility to biofilm formation depending upon the organism(s) present. This suggests that the type of implant material chosen for surgical procedures may affect the patient's wound healing response and clinical outcome, particularly when used in a contaminated environment.
\end{abstract}

\section{Introduction}

Prosthetic mesh materials have been widely used for decades in the diverse field of reconstructive surgery to improve surgical outcomes during incisional, inguinal, and ventral hernia repair, closure of large abdominal wall defects, and soft tissue and fascial reconstruction [1]. Hernia repair using mesh is one of the most common surgical procedures performed globally, with an estimated 20 million procedures performed worldwide per year [1]. Although complications have historically included recurrence, infection, fistula, and chronic pain, the development and widespread clinical use of modern synthetic polymers have reduced recurrence rates from over $40 \%$ to $15 \%$ or less [2-4]. Nevertheless, mesh infection continues to be a major concern, occurring in up to $12-16 \%$ of all ventral hernia repairs and often requiring reoperation [3-6]. In contaminated or potentially contaminated operative fields, infections are reported in up to $36 \%$ of procedures where synthetic materials are used [5-8].

A variety of surgical mesh materials currently exist, with the most common synthetics to include polypropylene, polyester, and polytetrafluoroethylene (PTFE) [1,9,10]. Modifications have been made to each of these materials in second- and third-generation prosthetics with the intent to reduce the inflammatory response, improve tissue ingrowth, and reduce infection rates [1,10,11]. Although numerous varieties are commercially available, no mesh material has demonstrated optimal biocompatibility and incorporation into host tissue $[1,12,13]$. We and others have shown in vivo that both chemical composition and filament structure are important mesh characteristics that may affect resistance to bacterial colonization, wound healing response, and ultimately the patient's clinical outcome [14-17].

Biofilms are a known important contributor to the infectious complications associated with prosthetic mesh implantation, causing substantial healthcare costs and excessive patient morbidity and mortality [18]. It is widely accepted that biofilms play a significant role in microbial persistence and infection chronicity $[19,20]$. Biofilms, one of the main causes of antibiotic treatment failure, are produced when populations of organisms coalesce and secrete an extracellular matrix composed of polysaccharides, proteins, and DNA that serves as a protective barrier against conventional antibiotic treatments and host defenses [21]. They are prevalent in at least $80 \%$ of all non-healing

${ }^{*}$ Correspondence to: Lisa M. Pierce, Department of Clinical Investigation, Tripler Army Medical Center, 1 Jarrett White Road, Honolulu, Hawaii, 96859, USA, Tel: (808) 433-5219; E-mail: lisa.m.pierce.civ@mail.mil

Key words: biofilm biomass, Candida albicans, methicillin-resistant Staphylococcus aureus, polymicrobial biofilm, Pseudomonas aeruginosa, synthetic mesh

Received: March 06, 2020; Accepted: March 23, 2020; Published: March 26, 2020 
chronic wounds and are common in patients with implanted medical foreign bodies or indwelling devices [18,22,23]. Mature biofilms are frequently polymicrobial, allowing for the inter-species transfer of metabolites and antimicrobial-resistance genes, thereby increasing overall virulence $[18,21]$. Implanted medical devices are often sites for bacterial-fungal interactions, and the organisms frequently isolated from these infections include Staphylococcus aureus (S. aureus), Pseudomonas aeruginosa ( $P$. aeruginosa), and Candida albicans ( $C$. albicans) [24]. In a recent study, polymicrobial biofilms were directly identified on mesh recovered from a series of patients experiencing infectious complications after ventral hernia repair using confocal microscopy, fluorescence in situ hybridization, and a novel molecular polymerase chain reaction-mass spectrometry-based assay to obtain a molecular profile of the biofilm organisms [18].

Previous studies have demonstrated in vitro that surface characteristics of prosthetic materials affect the ability of bacteria to attach and form a biofilm [25-30]. Although these investigations have demonstrated the formation of monomicrobial biofilms on various surgical meshes by different bacterial species in vitro, a paucity of data exists examining more clinically relevant polymicrobial biofilm formation by antibiotic-resistant organisms derived from patient wound specimens. Therefore, the aim of this study was to evaluate the ability of three clinical bacterial and fungal isolates to form mixed-species and single-species biofilms on five synthetic implant materials in vitro.

\section{Materials and methods}

\section{Implant materials}

Prosthetics used in this study included a lightweight monofilament polypropylene mesh (Prolene Soft, Ethicon Inc., Somerville, NJ), a lightweight monofilament polyester mesh (Parietex, Covidien, Mansfield, MA), a heavyweight multifilament polyester mesh (Parietex, Covidien), a mediumweight macroporous mesh constructed from PTFE fibers in a knitted design (Infinit Mesh, W.L. Gore \& Associates Inc., Flagstaff, AZ), and medical grade silicone sheeting (reinforced, 0.007 inch, gloss; BioPlexus Corp., Ventura, CA). The meshes were cut into uniform $1 \mathrm{~cm}^{2}$ pieces and autoclaved for sterilization.

These materials were chosen because they are commonly used in reconstructive surgical procedures including hernia repair. The evolution of polypropylene, polyester, and PTFE meshes by various manufacturers into composite materials is an ongoing process in order to improve patient outcomes and cost concerns of biologic prosthetics. For example, in 2017, GORE ${ }^{*}$ SYNECOR preperitoneal and intraperitoneal hybrid biosynthetic materials (W.L. Gore \& Associates), composed of macroporous PTFE knitted mesh and absorbable compounds, received $510(\mathrm{k})$ clearance from the U.S. Food and Drug Administration. The current study addresses the permanent synthetic mesh components of commercially available products.

\section{Microbes and growth conditions}

Clinical isolates of methicillin-resistant $S$. aureus (MRSA), multidrug-resistant $P$. aeruginosa, and C. albicans derived from wound specimens collected at Tripler Army Medical Center were prescreened using a crystal violet microtiter plate assay [31] to confirm their ability to form biofilms. Staphylococcus epidermidis (S. epidermidis) strain ATCC \#12228 was obtained from American Type Culture Collection (Manassas, VA).

Prior to experimental testing, bacterial and yeast stocks stored at $-80^{\circ} \mathrm{C}$ on cryobeads were serially passed twice on blood agar plates and incubated at $37^{\circ} \mathrm{C}$ to produce viable colonies. Isolated bacterial and yeast colonies were inoculated in tryptic soy broth and Sabouraud dextrose broth, respectively, adjusted to 0.5 on the McFarland turbidity scale, and then grown overnight at $37^{\circ} \mathrm{C}$ with shaking at $225 \mathrm{rpm}$. Overnight cultures were further diluted between 1:100 and 1:100,000 in brain heart infusion (BHI) broth with $5 \%$ bovine serum albumin (BSA) to give starting concentrations between $1 \times 10^{4}$ colony forming units $(\mathrm{CFU}) / \mathrm{ml}$ and $1 \times 10^{6} \mathrm{CFU} / \mathrm{ml}$. In an attempt to ensure establishment of polymicrobial biofilms with balanced populations, a higher inoculation ( 2 orders of magnitude) of C. albicans and MRSA compared to $P$. aeruginosa was used.

Mesh pieces were placed into individual wells of 24 -well tissue culture plates and were inoculated with $1 \mathrm{ml}$ of $\mathrm{BHI} / 5 \% \mathrm{BSA}$ containing approximately $1 \times 10^{6} \mathrm{CFU} / \mathrm{ml}$ MRSA, $P$. aeruginosa, or $S$. epidermidis for monomicrobial biofilms. For polymicrobial biofilms, additional meshes were inoculated with $1 \mathrm{ml} \mathrm{BHI/5 \%} \mathrm{BSA} \mathrm{containing}$ approximately $1 \times 10^{6} \mathrm{CFU} / \mathrm{ml} \mathrm{C}$. albicans, $1 \times 10^{6} \mathrm{CFU} / \mathrm{ml} \mathrm{MRSA}$, and $1 \times 10^{4} \mathrm{CFU} / \mathrm{ml}$ P. aeruginosa. Meshes were incubated at $37^{\circ} \mathrm{C} / 5 \% \mathrm{CO}_{2}$ for a total of 48 hours. Media in wells was aspirated and replaced with fresh BHI/5\% BSA after 24 hours. Uninoculated meshes incubated in $\mathrm{BHI} / 5 \% \mathrm{BSA}$ or phosphate buffered saline (PBS) were used as negative controls. Forty-eight-hour prosthetic-associated biofilms were then analyzed as described below.

\section{Crystal violet assay to assess biofilm biomass}

Biofilm biomass was determined in triplicate experimental replicates using a crystal violet assay [27,31]. Meshes were rinsed twice with PBS to remove non-attached planktonic bacterial or yeast cells and transferred to a new 24-well plate using sterile forceps. Meshes were fixed with $100 \%$ methanol for 15 minutes, air-dried, and incubated for 15 minutes with $0.1 \%$ crystal violet. Meshes were then washed thoroughly with water and air dried for approximately 1 hour. The crystal violet absorbed by the biofilm was released by the addition of $30 \%$ acetic acid for 15 minutes and the optical density was measured at 550 $\mathrm{nm}\left(\mathrm{A}_{550}\right)$ using a microplate reader (Synergy Multimode Reader HTX; Biotek Instruments, Winooski, VT). Measurements were normalized for each mesh by dividing the $A_{550}$ values of inoculated meshes by the mean of their respective $\mathrm{BHI} / 5 \% \mathrm{BSA}$ and $\mathrm{PBS}$ negative control (uninoculated) meshes and expressed as fold increase over control mesh.

\section{Fluorescence microscopy of polymicrobial biofilm-associated meshes}

Additional polymicrobial biofilm-associated meshes and uninoculated PBS control meshes (1 each per mesh type with the exception of silicone) were removed from each well, rinsed in 0.2 $\mu \mathrm{m}$ filter-sterilized water, and blotted gently to remove planktonic organisms. Meshes were transferred to a new 24-well plate and incubated for 30 minutes in $250 \mu \mathrm{l}$ staining solution containing $10 \mu \mathrm{g} /$ ml FilmTracer ${ }^{\mathrm{TM}} \mathrm{FM}^{*}$ 1-43 Green Biofilm Cell Stain (ThermoFisher Scientific, Waltham, MA) at room temperature and protected from light. The $\mathrm{FM}^{\circ} 1-43$ dye is a lipophilic styryl compound, is virtually nonfluorescent in aqueous media, and is believed to insert into the surface membrane where it becomes intensely fluorescent (excitation/ emission maxima 472/580 nm). Meshes were then rinsed in sterilefiltered water, transferred to a new 12- or 24-well plate containing filter-sterilized water, and viewed under an Olympus IX73 fluorescence microscope with a fluorescein isothiocyanate (FITC) excitation/ emission filter (Olympus America Inc., Center Valley, PA). Digital images were captured with cellSens imaging software using an Olympus DP80 camera. 


\section{Quantitation of viable organisms recovered from polymicrobial biofilms}

In a separate experiment, polymicrobial biofilm-associated meshes and uninoculated PBS control meshes (1 each per mesh type) were rinsed in PBS and blotted gently to remove planktonic organisms. Samples were then placed in $10 \mathrm{ml}$ sterile PBS in $15 \mathrm{ml}$ tubes, vortexed for 1 minute, and sonicated for 5 minutes in a Bransonic CPX2800H ultrasonic bath (40 kHz; Branson Ultrasonics Corp., Danbury, CT). Using the drop plate method with $4 \times 10 \mu \mathrm{l}$ spots per dilution, serial dilutions $\left(10^{1}\right.$ to $\left.10^{-8}\right)$ were plated onto selective agar (mannitol salt agar to identify MRSA, cetrimide agar to identify $P$. aeruginosa, and CHROMagar candida to identify C. albicans). Plates were grown 24 hours at $37^{\circ} \mathrm{C}$ before counting colonies. Growth of bacteria and $C$. albicans was expressed as CFU per mesh sample. The lower limit of detection was $1 \times 10^{3} \mathrm{CFU}$ per mesh.

\section{Statistical analysis}

Values were reported as means \pm standard error of the mean. A two-way analysis of variance followed by pairwise multiple comparison testing using the Holm-Sidak method was used to determine differences in biofilm biomass among the groups and to identify an interaction between material type and biofilm organism(s). Statistical analyses were performed using SigmaPlot 11.2 software (Systat Software Inc., San Jose, CA) with $\mathrm{p}<0.05$ considered significant.

\section{Results}

Crystal violet staining of biofilm biomass, CFU determination, and fluorescence microscopy revealed that all implant materials investigated in this study were capable of harboring biofilms, but that the relative abundance was dependent upon the particular material and organism(s) present (Figures 1 and 2; Table 1). A statistically significant interaction was identified between mesh type and biofilm type ( $p=0.02)$. Overall, PTFE meshes exhibited the highest levels of polymicrobial biofilm biomass, while multifilament polyester had the lowest levels (Figure 1). Polypropylene and polyester monofilament meshes supported similar biofilm growth. When comparing monomicrobial biofilms, $P$. aeruginosa demonstrated greater $(\mathrm{p}<0.05)$ biomass than MRSA and S. epidermidis on PTFE, polypropylene, and monofilament polyester. MRSA and S. epidermidis did not differ significantly among mesh types (Figure 1).

Viable bacteria and yeast cells were recovered from all polymicrobial biofilm-laden meshes after sonication at 48 hours (Table 1). Cell numbers in the polymicrobial biofilms ranged from $10^{4}-10^{5} \mathrm{CFU} C$. albicans, $10^{6}-10^{7} \mathrm{CFU}$ MRSA, and $10^{11}-10^{13} \mathrm{CFU}$ P. aeruginosa per $\mathrm{cm}^{2}$ mesh (Table 1). Although a higher inoculation ( 2 orders of magnitude) of $C$. albicans and MRSA compared to $P$. aeruginosa was used in order to facilitate cooperation of these species with $P$. aeruginosa, the $P$. aeruginosa was still able to dominate the 48-hour biofilms (5-7 orders of magnitude higher $\mathrm{CFU}$ counts than the other microbes). In contrast,

Table 1. Number of viable organisms recovered from 48-hour polymicrobial biofilmassociated surgical implant materials. No organisms were recovered from uninoculated meshes (PBS controls)

\begin{tabular}{|c|c|c|c|}
\hline Mesh material & $\begin{array}{c}\text { C. albicans } \\
\left(\mathrm{CFU} / \mathrm{cm}^{2} \text { mesh }\right)\end{array}$ & $\begin{array}{c}\text { MRSA } \\
\left(\mathrm{CFU} / \mathbf{c m}^{2} \text { mesh }\right)\end{array}$ & $\begin{array}{c}\text { P. aeruginosa } \\
\left(\text { CFU } / \mathrm{cm}^{2} \text { mesh }\right)\end{array}$ \\
\hline Silicone & $1.24 \times 10^{5}$ & $3.35 \times 10^{6}$ & $4.69 \times 10^{11}$ \\
\hline Multifilament Polyester & $4.73 \times 10^{5}$ & $1.66 \times 10^{7}$ & $1.00 \times 10^{12}$ \\
\hline Monofilament Polyester & $2.20 \times 10^{4}$ & $2.55 \times 10^{6}$ & $7.20 \times 10^{13}$ \\
\hline Monofilament Polypropylene & $2.34 \times 10^{4}$ & $2.85 \times 10^{6}$ & $1.55 \times 10^{12}$ \\
\hline Monofilament PTFE & $1.28 \times 10^{4}$ & $1.68 \times 10^{6}$ & $5.93 \times 10^{12}$ \\
\hline
\end{tabular}

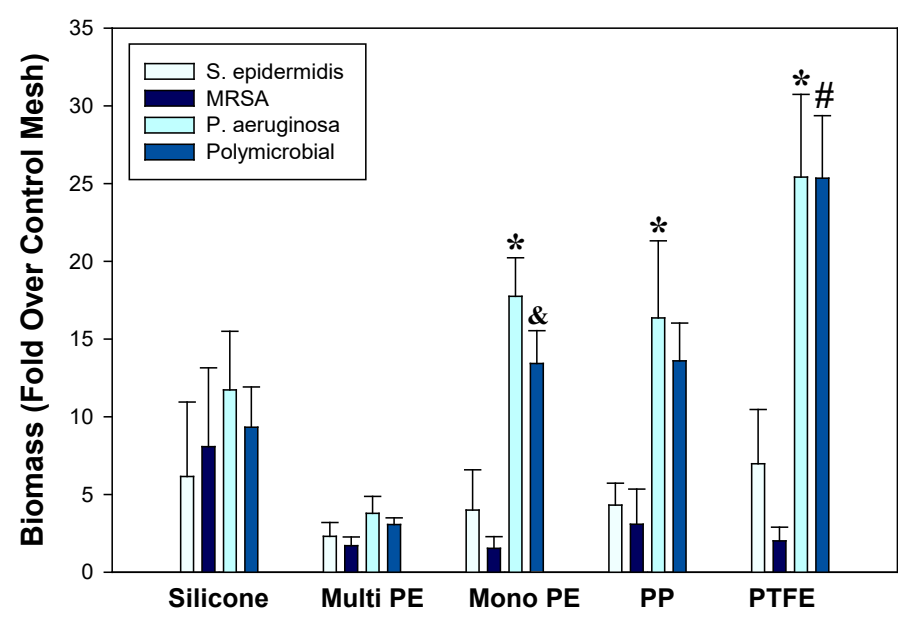

Figure 1. Monomicrobial and polymicrobial biofilm growth on various synthetic implan materials as determined by crystal violet staining of biofilm biomass. ${ }^{*} \mathrm{p}<0.05$ vs. MRSA and S. epidermidis; ${ }^{\mathrm{p}} \mathrm{p}<0.05$ vs. MRSA; ${ }^{\mathrm{p}}<0.05$ vs. MRSA and S. epidermidis. PE, polyester; PP, polypropylene.

the growth of C. albicans was somewhat suppressed by the bacteria (1-2 orders of magnitude lower than the initial inoculum). These results highlight the challenges of co-culturing different species in vitro due to the undesired killing of one or more species even though they may coexist in their natural environment [32].

Viability measurements by CFU counting of polymicrobial biofilms were not in complete agreement with biomass determinations by crystal violet assay. For example, overall CFU counts were not higher for PTFE than multifilament polyester and silicone, although biofilm biomass was significantly higher $(\mathrm{p}<0.05)$ for PTFE than these 2 materials (Table 1 and Figure 1). Nevertheless, visualization of $\mathrm{FM}^{*}$ 1-43-stained polymicrobial biofilm-associated meshes using fluorescence microscopy supported the biomass measurements and revealed more robust biofilm formation on PTFE compared to the other materials (Figure 2). Biofilm organisms and extracellular matrix could be seen as a hazy substance enveloping the mesh fibers (Figure 2). No staining was visualized on uninoculated meshes that were incubated 48 hours in PBS (not shown).

\section{Discussion and conclusion}

Most surgeons worldwide agree that the use of a prosthetic mesh is the preferred approach for hernia repair and other reconstructive surgical procedures despite the fact that the ideal mesh has not yet been developed [1]. Infection remains a challenge after implantation, often necessitating mesh removal [33]. Approximately 69\% of explanted meshes are caused by prosthetic infection [1]. It is feasible that unrecognized subclinical biofilm formation may be involved in mesh failure, resulting in a localized inflammatory response that may injure surrounding tissues and prevent mesh incorporation, ultimately leading to hernia recurrence [18].

Polymicrobial biofilms are an understudied and clinically relevant health problem, with the potential to harbor various organisms including bacteria and fungi $[18,19,21,24,30,32]$. Formation of polymicrobial biofilms on the surface of implants may be a major causative factor for antibiotic treatment failure and the development of infectious complications requiring reoperation [18]. Once a biofilm is established, it becomes difficult to eradicate the organisms present because the minimal antimicrobial concentration for eradication is difficult to achieve in vivo due to toxicity and limitations of renal and 

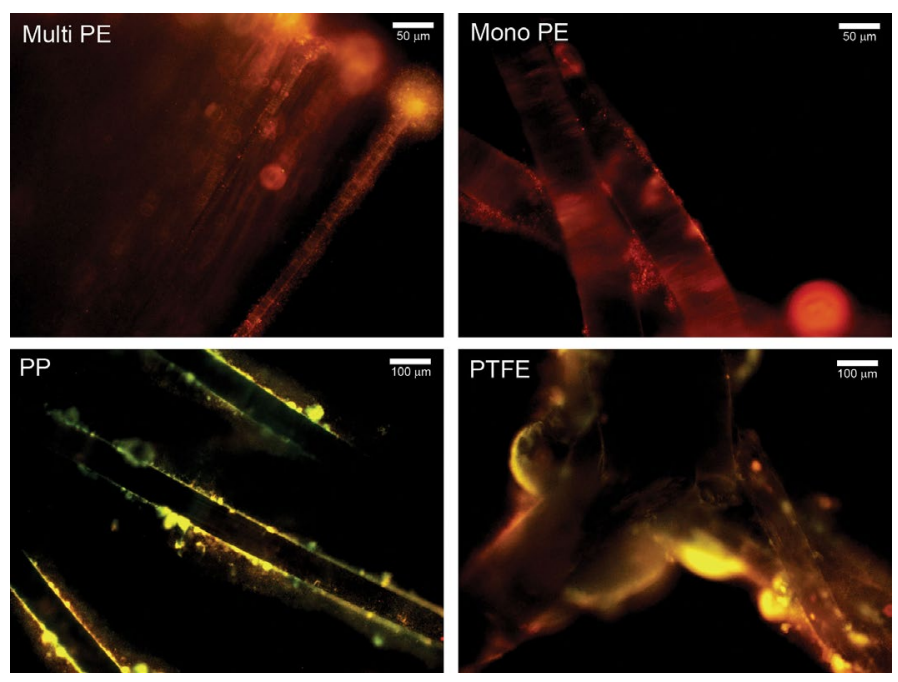

Figure 2. Representative fluorescent microscopy images of 48-hour polymicrobial biofilmassociated mesh fibers stained with $\mathrm{FM}^{\circledR} 1-43$. Biofilms were composed of MRSA, $P$ aeruginosa, and $C$. albicans. Mono PE, monofilament polyester; Multi PE, multifilament polyester.

hepatic functions [21]. Antibiotic tolerance for biofilm bacterial cells is reportedly 500-5000 times higher than for planktonic (free-floating) bacteria [21].

S. epidermidis and $S$. aureus are two of the most prevalent contaminating organisms associated with prosthetic infections because they are common skin commensal bacteria, are introduced easily into incisions leading to infection, and have the ability to form biofilms $[18,34]$. P. aeruginosa and C. albicans are also frequently isolated from infected implanted medical devices [19,24,27]. The number of organisms required to cause an infection in the presence of a prosthetic has been suggested to be approximately $1 / 10,000$ the number required to cause skin infections [29]. In this study we utilized an initial inoculation of $10^{6} \mathrm{CFU}$ or $10^{4} \mathrm{CFU}$ (in the case of $P$. aeruginosa in polymicrobial biofilms) to elicit an acute contamination of the meshes [34]. Although a higher inoculation (2 orders of magnitude) of C. albicans and MRSA compared to $P$. aeruginosa was used in order to facilitate cooperation of these species with $P$. aeruginosa in the polymicrobial biofilms, $P$. aeruginosa was clearly the outcompeting species.

Bacterial adhesion to biomaterials during implantation is critical to the development of mesh infection and subsequent biofilm formation. Several studies have shown that the roughness and material characteristics of the implant surface impact initial bacterial adherence [26-29]. Previous in vitro studies investigating synthetic meshes have determined that hydrophobicity, porosity, and filament number influence bacterial adhesion and monomicrobial biofilm formation by various Gram-positive and Gram-negative bacteria [26,28,29,34]. Bellows et al. [27] examined various biologic prosthetics in vitro and also showed that the formation of MRSA and P. aeruginosa monomicrobial biofilms is both material and species dependent. Overall, results from our study corroborated these findings, although MRSA and S. epidermidis monomicrobial biofilm biomass measurements were not significantly different among the 5 different synthetic mesh types investigated.

Unique to our investigation, we determined that all of the materials examined were capable of harboring C. albicans, MRSA, and $P$. aeruginosa polymicrobial biofilms, but that the relative abundance was dependent upon material type. We found that PTFE exhibited the highest levels of polymicrobial biofilm formation as detected by crystal violet staining of biofilm biomass and fluorescence microscopy (but not by CFU counts of viable organisms) compared to multifilament and monofilament polyester, monofilament polypropylene, and silicone sheeting. Interestingly, discrepancy between biomass and viability measurements has been observed in other studies [27]. The finding of enhanced polymicrobial biofilm formation on PTFE is in agreement with Engelsman et al. [28], who examined single-species biofilm growth in vitro by $S$. aureus, $P$. aeruginosa, and Escherichia coli on 6 synthetic surgical meshes. They discovered that PTFE, the most hydrophobic material tested, grew biofilms that were embedded in massive amounts of slime consisting of exopolysaccharide matrix and proteins. Englesman et al. [14] confirmed their results in an in vivo study and determined that hydrophobic PTFE meshes and multifilament meshes with a large surface area significantly increased bacterial persistence and spreading in the infected area, in contrast to monofilament polypropylene and lightweight meshes. Altogether, these results are consistent with clinical findings that PTFE meshes require removal in almost all cases of infection, while macroporous polypropylene meshes are salvageable in most cases [33].

Limitations of this study exist. Because this was an in vitro study, we were unable to evaluate the complex interactions between implant materials, microbes, and the host immune system. Future investigation will involve in vivo testing of polymicrobial biofilms using additional commercially available synthetic and biologic materials and will expand testing of unequal inocula combinations to ensure establishment of polymicrobial biofilms with balanced populations.

In conclusion, prosthetic-associated polymicrobial biofilms have significant clinical relevance. Findings from this study revealed that MRSA, $P$. aeruginosa, and C. albicans can cooperatively form mature biofilms on various surgical implant materials in vitro, and that different mesh constructs vary in their susceptibility to biofilm formation depending upon the organism(s) present. Macroporous PTFE mesh demonstrated increased vulnerability to polymicrobial and $P$. aeruginosa infection, suggesting that the type of implant material chosen for surgical procedures may affect the patient's wound healing response and clinical outcome, particularly when used in a contaminated environment.

\section{Acknowledgement}

The views expressed in this manuscript are those of the authors and do not reflect the official policy or position of the Department of the Army, Department of Defense, or the US Government.

\section{References}

1. Baylón K, Rodríguez-Camarillo P, Elías-Zúñiga A, Díaz-Elizondo JA, Gilkerson R, et al. (2017) Past, present and future of surgical meshes: a review. Membranes (Basel) 7: 47. [Crossref]

2. Shankaran V, Weber DJ, Reed RL, Luchette FA (2011) A review of available prosthetics for ventral hernia repair. Ann Surg 253: 16-26. [Crossref]

3. Satterwhite TS, Miri S, Chung C, Spain D, Lorenz HP, et al. (2012) Outcomes of complex abdominal herniorrhaphy: experience with 106 cases. Ann Plast Surg 68: 382388. [Crossref]

4. Kissane NA, Itani KM (2012) A decade of ventral incisional hernia repairs with biologic acellular dermal matrix: what have we learned? Plast Reconstr Surg 130: 194S-202S. [Crossref]

5. Carbonell AM, Criss CN, Cobb WS, Novitsky YW, Rosen MJ (2013) Outcomes of synthetic mesh in contaminated ventral hernia repairs. J Am Coll Surg 217: 991-998. [Crossref]

6. Rosen MJ, Krpata DM, Ermlich B, Blatnik JA (2013) A 5-year clinical experience with single-staged repairs of infected and contaminated abdominal wall defects utilizing biologic mesh. Ann Surg 257: 991-996. [Crossref] 
Colgan BA (2020) In vitro evaluation of Staphylococcus aureus, Pseudomonas aeruginosa, and Candida albicans polymicrobial biofilm growth on synthetic surgical implant materials

7. Birolini C, Utiyama EM, Rodrigues AJ Jr, Birolini D (2000) Elective colonic operation and prosthetic repair of incisional hernia: does contamination contraindicate abdominal wall prosthesis use? J Am Coll Surg 191: 366-372. [Crossref]

8. Darehzereshki A, Goldfarb M, Zehetner J, Moazzez A, Lipham JC, et al. (2014) Biologic versus nonbiologic mesh in ventral hernia repair: a systematic review and meta-analysis. World J Surg 38: 40-50. [Crossref]

9. Burger JWA, Luijendijk RW, Hop WCJ, Halm JA, Verdaasdonk EGG, et al. (2004) Long term follow-up of a randomized controlled trial of suture versus mesh repair of incisional hernia. Ann Surg 240: 578-585. [Crossref]

10. Matthews BD, Kercher KW (2002) Bioprosthetic materials in hernia repair. Probl Gen Surg 19: 7-13. [Crossref]

11. Cobb WS, Kercher KW, Heniford BT (2005) The argument for lightweight polypropylene mesh in hernia repair. Surg Innov 12: 63-69. [Crossref]

12. Leber GE, Garb JL, Alexander AI, Reed WP (1998) Long-term complications associated with prosthetic repair of incisional hernias. Arch Surg 133: 378-382. [Crossref]

13. Amid, PK (1997) Classification of biomaterials and their related complications in abdominal wall hernia surgery. Hernia 1: 15-21.

14. Engelsman AF, van Dam GM, van der Mei HC, Busscher HJ, Ploeg RJ (2010) In vivo evaluation of bacterial infection involving morphologically different surgical meshes. Ann Surg 251: 133-137. [Crossref]

15. Asarias JR, Nguyen PT, Mings JR, Gehrich AP, Pierce LM (2011) Influence of mesh materials on the expression of mediators involved in wound healing. J Invest Surg 24: 87-98. [Crossref]

16. Nguyen PT, Asarias JR, Pierce LM (2012) Influence of a new monofilament polyester mesh on inflammation and matrix remodeling. J Invest Surg 25: 330-339. [Crossref]

17. Cole WC, Balent EM, Masella PC, Kajiura LN, Matsumoto KW, et al. (2015) An experimental comparison of the effects of bacterial colonization on biologic and synthetic meshes. Hernia 19: 197--205. [Crossref]

18. Kathju S, Nistico L, Melton-Kreft R, Lasko LA, Stoodley P (2015) Direct demonstration of bacterial biofilms on prosthetic mesh after ventral herniorrhaphy. Surg Infect (Larchmt) 16: 45-53. [Crossref]

19. Harriott MM, Noverr MC (2011) Importance of Candida-bacterial polymicrobial biofilms in disease. Trends Microbiol 19: 557-563. [Crossref]

20. Clinton A, Carter T (2015) Chronic wound biofilms: pathogenesis and potentia therapies. Lab Med 46: 277-284. [Crossref]

21. Khatoon Z, McTiernan CD, Suuronen EJ, Mah TF, Alarcon EI (2018) Bacterial biofilm formation on implantable devices and approaches to its treatment and prevention. Heliyon 4: e01067. [Crossref]
22. Wu YK, Cheng NC, Cheng CM (2019) Biofilms in chronic wounds: pathogenesis and diagnosis. Trends Biotechnol 37: 505-517.

23. Wu H, Moser C, Wang HZ, Høiby N, Song ZJ (2015) Strategies for combating bacteria biofilm infections. Int J Oral Sci 7: 1-7. [Crossref]

24. Lohse MB, Gulati M, Johnson AD, Nobile CJ (2018) Development and regulation of single- and multi-species Candida albicans biofilms. Nat Rev Microbiol 16: 19-31. [Crossref]

25. An YH, Friedman RJ (1998) Concise review of mechanisms of bacterial adhesion to biomaterial surfaces. J Biomed Mater Res 43: 338-348. [Crossref]

26. Aydinuraz K, Agalar C, Agalar F, Ceken S, Duruyurek N, et al. (2009) In vitro $S$ epidermidis and $S$. aureus adherence to composite and lightweight polypropylene grafts. J Surg Res 157: e79-86. [Crossref]

27. Bellows C, Smith A (2014) In vitro study of biofilm growth on biologic prosthetics. Pol J Microbiol 63: 409-414. [Crossref]

28. Engelsman AF, van der Mei HC, Busscher HJ, Ploeg RJ (2008) Morphological aspects of surgical meshes as a risk factor for bacterial colonization. Br J Surg 95: 1051-1059. [Crossref]

29. Halaweish I, Harth K, Broome AM, Voskerician G, Jacobs MR, et al. (2010) Nove in vitro model for assessing susceptibility of synthetic hernia repair meshes to Staphylococcus aureus infection using green fluorescent protein-labeled bacteria and modern imaging techniques. Surg Infect (Larchmt) 11: 449-454. [Crossref]

30. Stoodley P, Sidhu S, Nistico L, Mather M, Boucek A, et al. (2012) Kinetics and morphology of polymicrobial biofilm formation on polypropylene mesh. FEMS Immunol Med Microbiol 65: 283-290. [Crossref]

31. Christensen GD, Simpson WA, Younger JJ, Baddour LM, Barrett FF, et al. (1985) Adherence of coagulase-negative staphylococci to plastic tissue culture plates: A quantitative model for the adherence of staphylococci to medical devices. J Clin Microbiol 22: 996-1006. [Crossref]

32. Thet NT, Wallace L, Wibaux A, Boote N, Jenkins ATA (2019) Development of a mixedspecies biofilm model and its virulence implications in device related infections. $J$ Biomed Mater Res B Appl Biomater 107: 129-137. [Crossref]

33. Warren JA, Love M, Cobb WS, Beffa LR, Couto FJ, et al. (2020) Factors affecting salvage rate of infected prosthetic mesh. Am J Surg: S0002-9610(20)30039-8. [Crossref]

34. Pérez-Köhler B, Sotomayor S, Rodríguez M, Gegúndez MI, Pascual G, et al. (2015) Bacterial adhesion to biological versus polymer prosthetic materials used in abdominal wall defect repair: do these meshes show any differences in vitro? Hernia 19: 965-973. [Crossref]

Copyright: (C2020 Colgan BA. This is an open-access article distributed under the terms of the Creative Commons Attribution License, which permits unrestricted use, distribution, and reproduction in any medium, provided the original author and source are credited. 